\title{
Tendencias, explicaciones y control policial frente a la violencia delictiva bajo el gobierno bolivariano
}

\author{
Trends, explanations and police control of violent crime \\ under bolivarian government
}

\author{
Luis Gerardo Gabaldón*
}

\begin{abstract}
Resumen: Los delitos violentos en Venezuela, en particular los homicidios, vienen aumentando sostenidamente desde la década de 1990, aunque de manera exponencial a partir de 1998, coincidiendo con la instauración de un gobierno de izquierda. Las explicaciones académicas usuales en Venezuela enfatizan la desigualdad, la tensión, la privación, el mercado de las drogas e incluso la impunidad como factores asociados, pese a que el gobierno chavista redujo sustancialmente la desigualdad, especialmente desde 2005, cuando los homicidios continuaron su ascenso, y promovió una reforma policial orientada a mejorar el desempeño técnico y a racionalizar el uso de la fuerza física. Esta aparente contradicción se discute en el contexto de una reforma policial inconclusa, de la ineficacia para controlar la disponibilidad y uso de las armas de fuego y de una creciente militarización de la seguridad ciudadana, que probablemente han contribuido al incremento situacional de la violencia, más allá de las políticas de reducción de la desigualdad social.
\end{abstract}

Palabras clave: Delincuencia. Policía. Política pública. Chavismo. Venezuela.

Abstract: Violent crime, particularly homicide, have sharply increased since the 1990's and exponentially since 1998, as a leftist government was established in Venezuela. Scholar explanations within the country mostly rely on inequality, strain, deprivation, drug markets and even impunity as main associated factors, although government successfully reduced inequality, especially since 2005 , while the crime rate soared, and promoted a police reform aimed to foster professionalism and moderate use of force. This contradiction is discussed in the context of an inconclusive police reform, a lack

* Doctor por la Universidad Andrés Bello (Caracas, Venezuela), especialista en Derecho Penal y Criminología (Universidad de Roma, Italia,. Es profesor titular de Derecho Penal y Criminología en la Universidad de Los Andes, Mérida, y en la Universidad Católica Andrés Bello, Caracas, Venezuela, y profesor visitante e investigador asociado de la Universidad de Nuevo México, Estados Unidos, durante 1991, 1992 y 1997 <luisgerardo gabaldon@gmail.com>. 
of control of firearms and increased intervention of the military in public safety issues, which probably have contributed to situational violence beyond policies for reduction of social inequality.

Keywords: Crime. Police. Public policy. Chavism. Venezuela.

\section{Indicadores de violencia delictiva en Venezuela en los últimos años}

Las tasas y delitos violentos en Venezuela vienen aumentando sostenidamente desde la década de 1990. Actualmente en Venezuela se cuenta con dos fuentes oficiales para el registro de homicidios a nivel nacional: la estadística delictiva, recogida por la policía de investigación (Cuerpo de Investigaciones Científicas, Penales y Criminalísticas, CICPC) y la estadística de salud (Ministerio de la Salud, MPPS). A continuación se presenta la evolución de los homicidios en términos de frecuencias absolutas y de la tasa por 100.000 habitantes en el septenio 2006-2012 según estas dos fuentes de datos.

Tabla 1. Totales y tasas de homicidio de las fuentes oficiales (CICPC y MPPS)

\begin{tabular}{lcccccccc}
\hline Organismo & $\begin{array}{c}\text { Indicador de } \\
\text { homicidio }\end{array}$ & $\mathbf{2 0 0 6}$ & $\mathbf{2 0 0 7}$ & $\mathbf{2 0 0 8}$ & $\mathbf{2 0 0 9}$ & $\mathbf{2 0 1 0}$ & $\mathbf{2 0 1 1}$ & $\mathbf{2 0 1 2}$ \\
\multirow{3}{*}{ CICPC } & Total & 12.557 & 13.156 & 14.589 & 13.985 & - & 11.185 & 14.007 \\
& Tasa & 46,7 & 48,2 & 52,6 & 49,7 & - & 38,8 & 47,8 \\
\multirow{3}{*}{ MPPS } & Total & 8.805 & 9.470 & 10.487 & 9.595 & 9.042 & 8.875 & 9.948 \\
& Tasa & 32,7 & 34,7 & 37,8 & 34,1 & 31,6 & 30,6 & 33,8 \\
\hline
\end{tabular}

Fuentes: Chacón, 2012, Fernández y Tabares, 2012, MPPS, 2010, 2011, 2012.

A partir de 2006, con ocasión de los diagnósticos de la Comisión para la Reforma Policial, se instauraron en Venezuela las encuestas nacionales de victimización con soporte del Instituto Nacional de Estadística y periodicidad trienal, lo cual facilitó la utilización de criterios muestrales precisos y la expansión de datos para la población según determinados dominios territoriales. Por primera vez se contemplaron registros de homicidios, una novedad a nivel internacional. Según estas estimaciones, la tasa nacional de homicidios ha aumentado desde 49,6 pcmh en 2006 a 75 pcmh en 2009, hasta 77,5 pcmh en 2012 (Gabaldón et al., 2007; INE 2010, 2013). 
Tabla 2. Totales y tasas de homicidio según las encuestas nacionales de victimización

\begin{tabular}{lccc}
\hline \multicolumn{1}{r}{ Indicador de homicidio } & $\mathbf{3}$ Años \\
\cline { 2 - 4 } Total (ine) & 13.368 & $\mathbf{2 0 0 9}$ & $\mathbf{2 0 1 2}$ \\
Tasa (ine) & 49,6 & 21.132 & 22.782 \\
\hline
\end{tabular}

Fuentes: Gabaldón et al, 2006, INE 2010, 2012.

Gráfico 1. Tasa de homicidio INE, CICPV y MPPS (2006, 2009 y 2012)

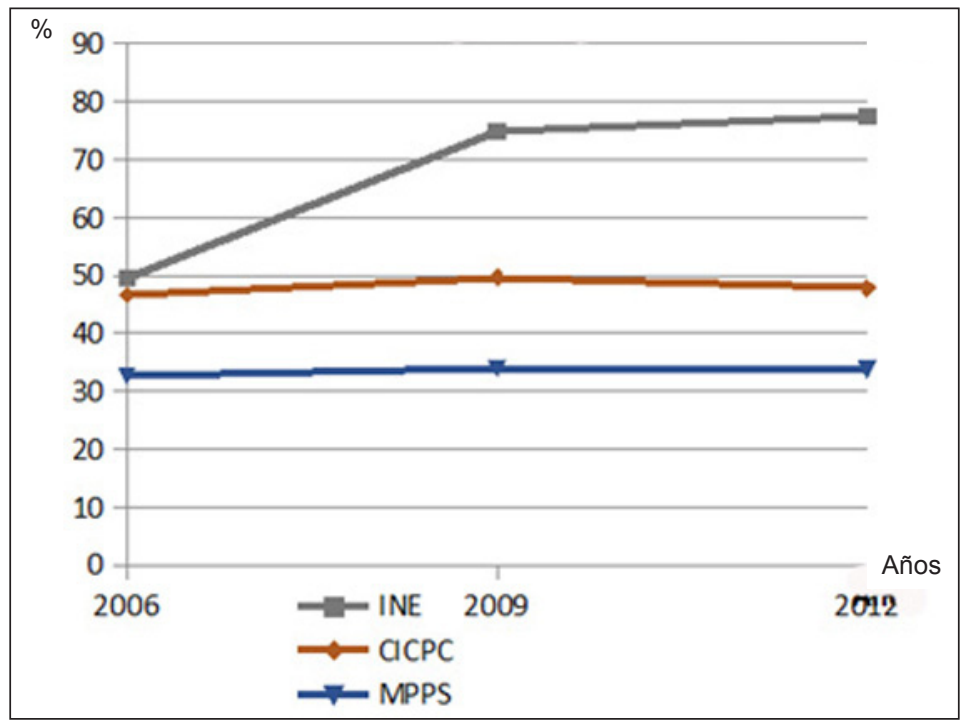

Fuentes: Gabaldón et al, 2006, INE 2010, 2012.

Según la última Encuesta Nacional de Victimización de 2012, 55\% de los homicidios se produjeron entre desconocidos y $96 \%$ con armas de fuego. Los hombres representaron el $77 \%$ de la víctimas y el $78 \%$ de los victimarios, mientras el grupo etario entre 15 y 29 años el $71 \%$ de las víctimas y el $47 \%$ de los victimarios (INE, 2013). La asociación de la violencia con las armas de fuego y la mayor vulnerabilidad masculina y juvenil está documentada también por los registros de salud: para 2008 95,7\% de las muertes ocasionadas por armas de fuego correspondieron a hombres y el grupo entre 18 y 44 años representó el 83\% de las víctimas masculinas y el 70\% de las femeninas (Chacón, 2012, 
p. 52-53). Los registros policiales muestran, además, consistencia con los datos de las encuestas de victimización; los homicidios con armas de fuego se concentraron para 2011 entre víctimas de 18 a 44 años en un en 75\%, conformando los hombres el 95\% de ellas (Fernández y Tabares, 2012, p. 92). Esto sugiere que los encuentros violentos letales, en los cuales la progresión de la agresión alcanza la liquidación física, están asociados a la masculinidad.

Las encuestas de victimización también han explorado la vulnerabilidad a la violencia según el estrato socioeconómico. En la encuesta de 2012, los grupos de pobreza extrema representaban el 15,6\% de la población y $9 \%$ de las víctimas de homicidio, mientras los sectores pobres, 53,9 y 61,6\%, en tanto que los más afluentes, $13 \%$ y 5,6\%, respectivamente, indicando estos hallazgos que no existe una relación estrictamente inversa entre estrato socioeconómico y violencia, como ha llegado a sugerirse.

Finalmente, en cuanto a variables situacionales, las encuestas nacionales han permitido determinar la concentración espacial y temporal de los homicidios. Para 2006, el 73\% ocurrió en el propio barrio o urbanización y el 94\% entre 6 de la tarde y 6 de la mañana (Gabaldón, Benavides y Parra, 2007), mientras para 2009 las proporciones fueron 62\% y 72\% (INE, 2010), y para 2012, 56\% y 64\%, respectivamente (INE, 2013).

Recientemente se ha planteado la cuestión de los funcionarios policiales muertos en actos de servicio o con ocasión de ellos. Si bien la literatura comparada sugiere que la policía no es inmune ni se comporta en forma ajena a la violencia social, y que el uso de fuerza policial tiende a incrementarse en zonas donde se registran más altos índices de violencia delictiva (Liska y Yu, 1992, p. 58; Worden, 1996, p. 37; Blumberg, 1997, p. 520), el abordaje de este problema en Venezuela es reciente. Un diagnóstico sobre condiciones asociadas al homicidio de 209 funcionarios policiales de distintos cuerpos del país ocurridos entre enero y noviembre de 2012 permite estimar que $45 \%$ de los casos fue vinculado al robo, aunque la sustracción del arma se produjo en $21 \%$ de los casos fuera de servicio y en $15 \%$ en servicio activo, cuando su porte es más visible, lo cual sugiere que el apoderamiento del arma podría haber sido un efecto colateral del homicidio y no la motivación principal de la muerte (CGP, 2012). Una investigación más reciente (Ávila, 2015b) sugiere mayor complejidad en la interpretación de la violencia contra la policía.

\section{Explicaciones sobre el incremento de la violencia}

Las explicaciones de la violencia delictiva entre los autores venezolanos tienden a ser eclécticas y relativamente gruesas. Algunos han propuesto diversidad de factores en cuanto al origen (falta de control familiar, decaimiento 
de control religioso y disparidades socioeconómicas), la promoción (desarrollo urbano irregular y exclusión, mercados de drogas, cultura de la masculinidad e impunidad judicial) y la facilitación de la violencia (disponibilidad de armas de fuego y presencia de vendedores al detal de estupefacientes, Briceño León, Villaveces y Concha Eastman, 2008), sin ponderación o factibilidad de verificación dentro un modelo teórico coherente. También se adelantó la hipótesis del deterioro institucional o del estado de derecho para explicar cambios en los homicidios dentro del país y en comparación con otros, sin que se hayan controlado las variaciones dentro de un mismo periodo o justificado los indicadores de deterioro institucional (Briceño León, 2011; 2012). Alguien sugirió la descentralización político administrativa en el país como disminución de la capacidad del Estado para controlar y prevenir la violencia, aunque no se presenta ningún indicador para sustentar esta conexión (Sanjuán, 2013). Estos marcos se han ampliado para incluir la noción de privación severa en la interpretación de la respuesta juvenil violenta (Zubillaga, 2007), asumiendo la violencia como expresión o reclamo frente a carencias (ver Katz, 1988) y recordando a las teorías de la tensión en criminología (ver Garrido, Stangeland y Redondo, 2001), dentro de una perspectiva cuya verificación y validación resultan problemáticos por falta de especificación de variables y condiciones asociadas a diversas modalidades de la violencia. En cuanto a las drogas, algunos no encuentran conexión entre consumo y comisión de delitos violentos (Crespo y Bolaños, 2008), mientras otros sostienen que los consumidores callejeros parecen estar más envueltos en pequeños hurtos y arrebatones, en tanto los actores violentos no parecieran participar directamente en el mercado ilegal, si bien la mayor disponibilidad de armas por el comercio internacional de las drogas, la corrupción policial y la aparición de riquezas súbitas podrían contribuir a la violencia criminal (Antillano y Zubillaga, 2014). En conjunto, las interpretaciones parecen identificar la privación severa como preponderante, dentro de una visión fundamentalmente negativa de la organización social y el sistema de justicia, frecuentemente descrito como ineficaz, inapropiado e injusto, lo cual pareciera ser un rasgo común en la perspectiva criminológica latinoamericana (Gabaldón, 2010b). Si bien las encuestas de victimización adelantadas desde 2006 han avanzado para identificar factores y variables asociados a la dinámica de la violencia interpersonal, tales como relaciones entre víctimas y victimarios, escalada de los conflictos, intensidad y expresividad de la violencia medida por heridas y daños, desplazamiento y vecindad o cercanía entre víctimas y victimarios, se requiere más investigación para proponer una discusión sustentada que soporte las políticas públicas de contención de la violencia. 


\section{La reforma policial: desarrollo, logros y desafíos entre 2006 y 2012}

La Comisión Nacional para la Reforma Policial fue instalada el 10 de abril de 2006. Entre los antecedentes inmediatos cabe destacar los escándalos de participación policial en graves hechos delictivos durante los seis meses anteriores. Se abordaron varios diagnósticos, consultas y propuestas que implicaron a más de 70.000 personas durante un periodo de 9 meses. Sus productos constituyeron la base para lo que se ha denominado el nuevo modelo policial, concretado en las leyes que gobiernan la estructura y estatuto funcional de los cuerpos de policía ostensiva del país desde 2008, y de los cuerpos de policía de investigación desde 2012. A diferencia de lo ocurrido en otros países (Gabaldón y Antillano, 2008), el cambio promovido para la policía no era una cuestión de imagen sino de desempeño, lo cual indica que la presencia y reconocimiento simbólico de la policía en Venezuela no es tan relevante como en otras sociedades.

El proceso de implementación de la reforma ha sido complicado $\mathrm{y}$ signado por los vaivenes de la política. Se requirió la generación de un espacio de confianza y legitimidad para su instrumentación, complicada debido a la polarización y el proceso electoral de 2006, así como el desarrollo de la normativa institucional que correspondió al Consejo General de Policía, órgano ministerial de apoyo y asesoría, lo cual funcionó razonablemente entre 2009 y 2012. Sin embargo, el giro que ha tomado la gestión ministerial a partir de 2014 sugiere una política de minimización y relativización de la dimensión técnico institucional a favor de un modelo militarizado que contraviene las bases mismas del nuevo modelo policial.

En materia del uso de la fuerza física por parte de la policía, cuestión alrededor de la cual se han centrado las críticas históricas sobre el desempeño policial venezolano (Birkbeck y Gabaldón, 2003; Provea, 2013; Red de Apoyo por la Justicia y por la Paz, 2010), la nueva legislación ha contemplado la adopción de escalas progresivas para el uso de la fuerza en función de la resistencia y oposición del ciudadano y la limitación del uso de la fuerza mortal para la defensa de la vida del funcionario o de un tercero. Para 2012, 9.000 cursantes del Plan Único de Formación Policial de la Universidad Experimental de la Seguridad recibían entrenamiento en uso diferenciado y progresivo y de la fuerza, y dicho programa se pretendía extender a 5.000 miembros del cuerpo de investigaciones científicas, penales y criminalísticas. De las denuncias acumuladas por violación de derechos humanos en el decenio 2000-2009, el 20\% corresponde a ejecuciones o alegados enfrentamientos 
por parte de la policía (autos de resistencia), lo cual sugiere una significativa incidencia de fuerza física excesiva (Red de apoyo por la justicia y por la paz, 2010, p. 23), si bien la implementación de la reforma policial parece haber reducido su proporción entre las policías uniformadas, que han sido las destinatarias primarias de los nuevos estándares; una comparación entre 2008 (fecha de promulgación de la nueva ley) y 2013, muestra que las violaciones del derecho a la vida se redujeron de $46 \%$ a $15,6 \%$ entre las policías de los estados y de $17 \%$ a $10 \%$ entre las policías uniformadas, mientras se incrementaron de $19,1 \%$ a $48 \%$ para el cuerpo de investigaciones científicas, penales y criminalísticas (Provea, 2013, p. 456).

Otro importante aspecto de la reforma ha consistido en la auditoría y rendición de cuentas mediante una combinación de controles internos y externos. Dentro de los últimos, la ley del estatuto de la función policial (Venezuela, 2009) previó en el art. 84 los comités ciudadanos de control policial, integrados por 5 personas en la cabecera de la jurisdicción de despliegue de cada cuerpo de policía, con la función de hacer seguimiento del desempeño en cuanto a gestión administrativa, funcional y operativa. Una evaluación sobre el proceso de facilitación y conformación de estos comités entre enero 2011 y julio 2012, permitió identificar elementos que podrían comprometer su eficacia e imparcialidad, destacándose la injerencia de representantes políticos de alcaldías, gobernaciones y otras instituciones gubernamentales, además de la propia policía, así como el sesgo partidista, dado que gran parte de los miembros que conforman los CCCP forman parte de los consejos comunales, instancias con clara posición afecta al partido del gobierno, de origen clientelar. Otra posible amenaza son los vínculos de delincuentes con integrantes de los comités y con los cuerpos de policía, incluyendo la amenaza y la manipulación (Gabaldón et al., 2014). Aunque desde 2002 el gobierno ha promovido los consejos comunales como la expresión más auténtica de la cogestión participativa, ellos no excluyen a otras instancias sociales estructuradas. La investigación disponible muestra que los consejos comunales parecen mostrar poco interés, gran confusión y poca articulación en materia de seguridad pública (Machado, 2008; Monsalve y Munévar, 2014).

\section{Desarme}

En mayo de 2011 se instaló la Comisión presidencial para el control de armas, municiones y desarme, presidida por el Ministro del Interior y Justicia, con participación de representantes del ejecutivo nacional, la Asamblea Nacional, el Ministerio Público, los cuerpos policiales, el sector académico y organizaciones privadas y de la sociedad civil. Se preparó un 
amplio programa de diagnóstico, consulta y divulgación que ha concluido con estudios cuantitativos y reportes de consultas entre grupos diversos. Se preparó una ley, aprobada en 2013 (Venezuela, 2013a), varias resoluciones ministeriales sobre control y restricción de armas y municiones y se lanzó una costosa campaña publicitaria a través de figuras mediáticas para posicionar violencia asociada a las armas de fuego. Entre los estudios destacan tres encuestas en parroquias caraqueñas para evaluar uso de armas de fuego en actos delictivos y mediante exhibición simbólica entre 2010 y 2012 (Gabaldón, Benavides y da Rocha, 2012). Dichas encuestas confirmaron resultados previos: predominancia de hombres y jóvenes como víctimas en el uso de las armas de fuego, victimización de estratos I y II (más altos) contra la propiedad y IV (más bajos) contra las personas; victimarios predominantemente entre grupos jóvenes de edad y hombres, con uniformidad inter parroquial. Casi $90 \%$ de los encuestados consideraron riesgoso poseer armas de fuego en el hogar, concordando las mujeres más con esta apreciación que los hombres, y casi $25 \%$ de los encuestados refirieron casos de heridas por balas perdidas, lo cual sugirió alto riesgo de lesividad aleatoria por el uso de dichas armas.

La ejecución de las previsiones de la ley para el desarme de 2013 no han sido consistentes y sostenidas. Los esfuerzos gubernamentales parecen haberse dirigido al despliegue publicitario de la inconveniencia de las armas de fuego y a la celebración de actos rituales de destrucción de armas de bajo calibre o artesanales, mientras crecientemente, individuos y grupos exhiben en forma ostensiva armas poderosas que podrían provenir de la propia dotación militar del estado. Por otro lado, las encuestas de victimización muestran que las armas de fuego se utilizan con mayor frecuencia contra desconocidos, lo cual es muy claro para el delito de robo, que representa el $64 \%$ de la victimización total y se comete en un $88 \%$ de los casos con armas de fuego (INE, 2013). Esto indica que las iniciativas centradas en el control y minimización de riesgos dirigidos hacia personas estrechamente relacionadas podría ser un esfuerzo desorientado. Si bien los resultados de las encuestas parroquiales indican que la percepción de riesgo por posesión de armas de fuego es elevada entre la población, y que ello podría constituir una ventaja comparativa para apoyar planes de desarme, esto no implica delegación estatal de la responsabilidad por la supervisión de los planes de desarme en las comunidades, como parecieran sugerir algunas acciones gubernamentales recientes (El Nacional, 2014b).

La cuestión de las armas de fuego en manos de la población no fue definida como problema en ninguno de los planes de la nación en los últimos cuarenta años, incluyendo los planes de gobierno o los discursos del presidente Chávez (Gabaldón, 1987; Monsalve y Gabaldón, 2014). Ahora se sabe que la 
asociación entre homicidios y armas de fuego es muy estrecha, aunque desde hace tiempo se conocía la mutación violenta de los delitos contra la propiedad debido a la disponibilidad de armas de fuego (Bettiol, 1983). El problema ha resurgido en forma dramática debido a la circulación de información en tiempo real, a la publicidad, a la exhibición y a la utilización de armas de fuego en situaciones de cotidianidad, banalidad, ensañamiento o desafío a la autoridad que producen escándalo. Sin embargo, el aumento de la delincuencia (Birkbeck, 2004) y la polarización política (Antillano, 2012) no favorecen un ambiente de acuerdos mínimos para contener y reducir la disposición a armarse por parte de la población y la nueva ley para el desarme podría carecer de impacto efectivo. Más allá de la normativa legal es preciso un consenso básico sobre la aplicación del programa de desarme con criterios de eficacia, transparencia, y consistencia.

\section{Militarización de la seguridad pública}

Según el art. 332, ap. 1 de la Constitución de 1999, los órganos de seguridad ciudadana son de carácter civil. Sin embargo, los distintos componentes militares de la Fuerza Armada Nacional han venido interviniendo en forma extensa en el patrullaje y despliegue policial de vigilancia, al menos desde 2004, en forma prácticamente rutinaria (Gabaldón, 2007, p. 93). Más recientemente, con la aprobación legislativa del denominado "Plan de la Patria", o Segundo Plan Socialista de Desarrollo Económico y Social de la Nación 2013-2019, se estableció como objetivo estratégico general el incremento de la capacidad defensiva del país y de la actividad cívico militar para neutralizar el crimen transnacional y la acción de grupos generadores de violencia, así como la participación activa del pueblo para consolidar la unión cívico militar (Venezuela, 2013b, p. 60). La confusión entre la seguridad de la nación (amenazas externas) con la seguridad ciudadana (control de vulnerabilidad y riesgo interno) se ha venido acentuando, aunque dicha propuesta tiene raíces en la insurgencia militar de 1992 que, según algunos análisis recientes podría ser independiente, incluso, de la tendencia izquierdista que se consolidó con Chávez a raíz de las elecciones de 1998 (Rivero, 2010, p. 224-250), habiendo indicios de que las presiones militaristas descalificadoras del gobierno civil que se manifestaron desde la guerra de independencia cobraron una dimensión seudo tecnocrática después del gobierno democrático instaurado luego de la dictadura de Pérez Jiménez en 1958 (Asuaje et al., 2007). Con motivo de las protestas y manifestaciones callejeras agudizadas desde 2013, la intervención militar se ha hecho masiva y ha dado lugar a polémica, denuncias nacionales e internacionales y a la aprobación de instructivos para el uso de armas de fuego 
en manifestaciones, cuestionados en cuanto trasladarían al ámbito militar el despliegue de la fuerza física y las tácticas de control de dichas manifestaciones, lo cual es una competencia policial de naturaleza civil (Provea, 2013). Probablemente debido al perfil de quienes han manifestado públicamente en los últimos tiempos, estudiantes y miembros de las clases medias, así como a la extensión de este fenómeno y la producción de muertes en las calles que no se veían desde 2002, ha resurgido una fuerte polarización política y la percepción gubernamental de que se trata de acciones desestabilizadoras orientadas desde el exterior que procurarían la caída del gobierno.

La extensión progresiva del control militarizado a la seguridad pública se manifiesta también en la designación cada vez más frecuente de altos rangos militares para dirigir las policías, contraviniendo lo establecido en el art. 6 de la ley orgánica del servicio de policía y cuerpo de policía nacional bolivariana (Venezuela, 2008). Incluso el denominado plan de patrullaje por cuadrantes, que se viene aplicando desde 2012, fragmenta la decisión de respuesta a emergencias y despoja a los comandos naturales de la policía civil de la coordinación y supervisión en la prestación del servicio, reproduciendo un modelo militar de ocupación de zonas cuyas manifestaciones más dramáticas han ocurrido desde julio de 2015, mediante las llamadas operaciones de liberación del pueblo, que procuran saturación oportunista, masiva y violenta de áreas residenciales populares con una lógica de ocupación de un espacio enemigo, contrariando los propios propósitos de la reforma policial en el sentido de fomentar el acatamiento y el sometimiento pacífico de la población a la autoridad policial, y que arrojaron como resultado, en algo más de dos meses siguientes a su aplicación, un saldo de 106 muertos entre sospechosos o sindicados de delitos (Ávila, 2015a). Estas operaciones han continuado en 2016 y sus partes de resultados se concentran en el número de delincuentes abatidos en diversas zonas populares, predominantemente urbanas, del país.

Debe destacarse que si bien la guardia nacional, desde su establecimiento en 1937 ha intervenido en forma principal y complementaria en funciones de vigilancia, protección y orden público (Gabaldón, 1999), la nueva Constitución define la seguridad ciudadana como una cuestión de naturaleza civil. Siendo la guardia nacional un componente de la Fuerza Armada Nacional, su intervención en materia de orden interno, considerando la naturaleza militar de dichas operaciones, conforme a lo establecido en el numeral 2 del art. 42 de la ley orgánica de la Fuerza Armada Nacional, debería ser subsidiaria. La utilización rutinaria de la Fuerza Armada termina produciendo una relativa insensibilización por parte de la población, inconveniente por cuanto desde un punto de vista estrictamente táctico el uso de escalas progresivas y 
diferenciadas para la fuerza policial, inadecuadas en la lógica militar de la saturación e impacto máximo, procura incrementar el acatamiento evitando la escalada de la situación de confrontación mediante el nivel de coacción más bajo posible (Birkbeck y Gabaldón, 2003, p. 132).

\section{Conclusiones y perspectivas}

La información y fiabilidad de las tasas de ocurrencia delictiva violenta y de las variables demográficas y situacionales asociadas a la victimización es una tarea prioritaria para un subcontinente que ha sido definido como la región más violenta del mundo (OPS, 2002; Pnud, 2013). El avance hacia registros más confiables y estandarizados requerirá iniciativas a nivel de los gobiernos y agencias gubernamentales, muchas veces refractarios a cambios y sugerencias debido a la inercia institucional y al efecto político adverso que representan las elevadas tasas de delitos violentos. También requerirá la promoción de investigación y sistemas de registro alternativo, mediante encuestas de victimización y estudios de casos, con el fin de generar datos que permitan la comparación y la adopción de políticas basadas en estimaciones realistas e indicadores susceptibles de verificación, seguimiento, evaluación e intervención dentro del marco de políticas públicas concertadas de seguridad pública. La violencia social no es, por otra parte, independiente de la violencia policial. Una evaluación reciente sobre una muestra de 162 egresados del Plan Nacional de Formación Policial y sobre 387 oficiales del cuerpo de policía nacional bolivariana desplegados en los estados centrales del país, sugiere que ha habido gran énfasis en los programas de instrucción y entrenamiento en el diálogo y la conciliación como parte de los niveles más bajos de la escala, relativos a la presencia y aproximación sin contacto físico, mientras las capacidades de tolerancia y comunicación con los ciudadanos son frecuentemente mencionadas como lo más importante aprendido en el centro de formación o en el cuerpo policial (Gabaldón, 2016). Sin embargo, los datos sugieren que el entrenamiento efectivo podría no abarcar el espectro completo en el uso de la fuerza, estimulando pasividad y escasa respuesta frente a la victimización delictiva, lo cual podría contribuir a reforzar la escalada de situaciones de amenaza y riesgo y al incremento de la violencia asociada a la incertidumbre y falta de control de las contingencias (Gabaldón, 2010a), como pareciera estar sucediendo con las denominadas operaciones de liberación del pueblo.

En cuanto a las políticas públicas de control delictivo, se observa falta de selectividad y focalización en las medidas de control de la criminalidad, lo cual pareciera guardar relación con una visión globalizadora, indiscriminada y errática en la aproximación a la delincuencia. Ello se aprecia en los discursos 
presidenciales y los programas de gobierno propuestos desde 1999, cuando el abordaje del problema se hizo inespecífico, aleatorio y desinformado de los datos y de la literatura vinculados a las tendencias y explicaciones sobre la delincuencia (Monsalve y Gabaldón, 2014, p. 72-79). Se observa también en la actividad policial y judicial que no parecieran orientarse a dar la relevancia debida a los hechos de violencia; una estimación para 2011 muestra que las detenciones policiales por casos conocidos de homicidio representaron sólo $19 \%$ y que los expedientes remitidos al Ministerio Público no superaban el $6 \%$, siendo estos casos remitidos inferiores a los correspondientes a las lesiones personales y a la violencia doméstica (Gabaldón, 2013, p. 233). Esto sugiere que la policía judicial presta mayor atención a la resolución de lesiones personales y violencia contra la mujer que a los homicidios, lo cual pareciera absurdo, aunque en el poder policial de negociación del caso con los imputados antes de la notificación al Ministerio Público podría radicar la verdadera razón de esta aparente paradoja (Antillano et al., 2012).

Se observa en las políticas oficiales una falta de seguimiento y medición de efectos, así como discontinuidad, incluso dentro del periodo de un solo gobierno, aunque este rasgo no es exclusivo del gobierno instaurado a partir de 1999 (Monsalve y Gabaldón, 2014). A ello se agrega la falta de transparencia, publicidad y difusión de registros e incluso estudios promovidos por el propio gobierno, como lo ilustran los casos de los informes finales de la Comisión para la Reforma Policial, en 2007 (Birkbeck y Gabaldón, 2009, p. 181-182) y, más recientemente, la Encuesta nacional de victimización y percepción de seguridad ciudadana (2012) por resultar sus datos desfavorables al gobierno en cuanto a incremento de la delincuencia violenta. Todo ello dificulta el seguimiento y medición de efectos de cualquier política con datos oficiales, mientras se complica emprender estudios independientes que pueden resultar muy costosos y no contar con información oficial que contribuya a validar otros resultados. A largo plazo, el efecto es una política errática, que se reinventa día a día con planes intuitivos u oportunistas y que impide la adopción de líneas de acción sostenidas en el tiempo, incluso por un mismo gobierno, situación reforzada por la continua rotación de los ministros y la falta de una burocracia técnica especializada y estable en las instancias encargadas de recabar, procesar y analizar los datos sobre la criminalidad.

Finalmente, pareciera haberse acentuado en Venezuela una promiscuidad entre la coacción pública y privada, resultando la primera aleatoria y recesiva, y la segunda emergente, incluso más predecible, aunque informal y cruda (Gabaldón, 2015; Antillano, 2015). En la contemporaneidad se asume como deseable restringir la coacción al ámbito público, estatal, escrutable, regulado, 
sujeto a alguna forma de rendición de cuentas (Gabaldón, 2000, p. 210). En cuanto a la coacción legal representada por el derecho penal, algunos han considerado que el carácter público del castigo es un rasgo irreversible de la cultura actual a nivel planetario (Garland, 1999, p. 335-337). El ejercicio de la fuerza debería estar sometido a protocolos de actuación, a la legalidad, a la sujeción a directrices y a escalas progresivas y diferenciadas. La formalidad constituiría la justificación y límite de la violencia estatal, representando un signo de división del trabajo propia de las sociedades contemporáneas: solamente mediante un conocimiento técnico y especializado se estaría en condiciones de aplicar la fuerza estrictamente necesaria para lograr el objetivo legítimo que se persigue. La situación venezolana pareciera ser la de un círculo vicioso donde la eficacia simbólica de la coacción se diluye, aumentando la rebeldía y resistencia de la población (Antillano, 2010, p. 342-343). La eliminación de la detención policial preventiva por el Código Orgánico Procesal Penal en 1998 y por la Constitución en 1999, podría haber sido un factor de estímulo al ejercicio de la violencia policial como expresión de castigo y control (Birkbeck y Gabaldón, 2009, p. 182; Monsalve Briceño, 2006). Por otra parte, la facilidad de organizarse que tienen diversos actores para definir espacios alternos al Estado, donde el criterio de la informalidad en la aplicación de la fuerza toma primacía, constituye una cuestión a evaluar en el momento actual. Ello podría ayudar a entender la ocupación de espacios que se sustraen a un control, consenso o acuerdo público, incluyendo partícipes que no convienen, pero se resignan, a la aplicación de reglas impuestas de manera informal y oportunista, debido a la heterogeneidad, fragmentación o incapacidad de coaliciones eficientes. La expansión de la violencia privada o privatizada por el mismo Estado, cuando permite grupos armados dentro o fuera de zonas de paz o despeje (El Nacional, 2014a), podría generar, al final, una escalada de la violencia del Estado cuando se procura restringir lo que en un primer momento se dejó expandir, y la reocupación de esos espacios se convierte en una consigna política y un programa de acción militar y policial rudo y hasta atroz, con tácticas propias de campañas bélicas y con blancos designados, antes que en una política sostenida para el control se la delincuencia y el mantenimiento de la seguridad pública. Estas campañas plantean un serio desafío para la gobernanza, el control social y la reducción de la violencia política y delictiva en la Venezuela contemporánea.

\section{Referencias}

ANTILLANO, Andrés. Cuando los presos mandan: control informal dentro de la cárcel venezolana. Espacio Abierto, v. 24, n. 4, p. 16-39, 2015. 
ANTILLANO, Andrés. Seguridad y política en la Venezuela bolivariana: la seguridad en el debate político venezolano entre 1998 y 2009. Espacio Abierto, v. 21, n. 4, p. 701-726, 2012.

ANTILLANO, Andrés. ¿Qué conocemos de la violencia policial en Venezuela? Las investigaciones e hipótesis sobre el uso de la fuerza física por la policía. Espacio Abierto, v. 19, n. 2, p. 331-345, 2010.

ANTILLANO, Andrés; ZUBILLAGA, Verónica. La conexión drogas ilícitas y violencia: una revisión de la literatura y consideraciones a la luz de la experiencia venezolana. Espacio Abierto, v. 23, n. 1, p. 129-148, 2014.

ANTILLANO, Andrés; POJOMOVSKI, Iván; BASTARDO, Alejando; TORRES Ivonne; PALÍS, Iván. Qué hace el CICPC: descripción de las actividades rutinarias de investigación de un grupo de detectives. In: Luis Gerardo Gabaldón; Soraya El Achkar (orgs.). Diagnóstico y propuestas de reforma del cuerpo de investigaciones cientificas, penales y criminalísticas. Caracas: Consejo General de Policía, 2012. p. 39-53.

ASUAJE, Manuel; SERRITIELLO, Américo; PICCARDO, Antonio; GONZÁLEZ, Pausides. De militares para militares. Caracas: El Perro y la Rana, 2007.

ÁVILA, Keymer. Si sacas el ejército a la calle estás matando al modelo policial, 2015a $<$ contrapunto.com/noticia/keymer-avila> (26 nov. 2015).

ÁVILA, Keymer. Muertes violentas de policías en Caracas: estudio de casos de funcionarios de seguridad víctimas de homicidio. Espacio Abierto, v. 24, n. 4, p. 40-66, 2015.

BETTIOL, Daniela. Modalidades en los delitos contra la propiedad en Venezuela, 1930-1983. Revista Cenipec, v. 8, p. 73-106, 1983.

BIRKBECK, Christopher. Experticia y prevención del delito: un estudio comparado de Canadá y Venezuela. Capítulo Criminológico, v. 32, n. 2, p. 147-189, 2004.

BIRKBECK, Christopher; GABALDÓN, Luis Gerardo. Venezuela: policing as an excersise in authority. In: Mercedes S. Hinton; Tim Newburn (orgs.). Policing developing democracies. New York: Routledge, 2009. p. 165-187.

BIRKBECK, Christopher; GABALDÓN, Luis Gerardo. Conclusión: La definición de los usos justificados de la fuerza en el desempeño policial: propuestas para un proyecto de investigación comparada. In: Luis Gerardo Gabaldón; Christopher Birkbeck (orgs.). Policía y fuerza física en perspectiva intercultural. Caracas: Nueva Sociedad y Ministerio de Ciencia y Tecnología, 2003. p. 125-137.

BLUMBERG, Mark. Controlling police use of deadly force: assessing two decades of progress. In: Roger G. Dunham; Geoffrey P. Alpert (orgs.). Critical issues in policing: contemporary readings. Prospect Heights: Waveland Press, 1997. p. 507-530.

BRICEÑO LEÓN, Roberto. La comprensión de los homicidios en América Latina ¿pobreza o institucionalidad? Ciência e Saúde Coletiva, v. 17, n. 12, p. 3159-3170, $2012<10.1590 /$ S1413-81232012001200002>.

BRICEÑO LEÒN, Roberto. Las tres fases de la violencia contemporánea en Venezuela. Debates Iesa, v. 16, n. 3, p. 14-19, 2011.

BRICEÑO LEÓN, Roberto; VILLAVECES, Andrés; CONCHA EASTMAN, Alberto. Understanding the uneven distribution of homicide incidence in Latin America. International Journal of Epidemiology, v. 37, n. 4, p. 751-757, $2008<10.1093 / \mathrm{ije} /$ dyn153>. 
CGP, Consejo General de Policía. Caracterización de los funcionarios policiales fallecidos por homicidio en Venezuela, Enero-Noviembre 2012. Caracas: Consejo General de Policía, 2012. p. 39.

CRESPO, Freddy; BOLAÑOS, Mireya. Delitos violentos: entre los vapores de la drogadicción. Capítulo Criminológico, v. 36, n. 3, p. 101-141, 2008.

CHACÓN, Andrea. Muertes ocasionadas por el uso de armas de fuego (MPPS). Sentido e impacto del uso de armas de fuego en Venezuela. Caracas: Comisión Presidencial para Control de Armas, Municiones y Desarme, 2012. p. 39-67<www. comisionpresidencialdesarme.gob.ve>.

EL NACIONAL. Delincuentes controlan “zonas de paz” en Barlovento, p. 8, 19-12014a <www.el-nacional.com/sucesos/Acevedo-Andres_Bello-Barlovento-Burozextorsiones-homicidios-Miranda-Paz-robos-secuestros-tiroteos-VERSIONFINALzonas_de_paz_0_339566162.html> (25 nov. 2016).

EL NACIONAL. Gaby Arenas de Meneses: "Solo los delincuentes ganan con canje de armas por dinero". p. 4, 2-2-2014, 2014b <www.el-nacional.com/politica/GabyArenas-Meneses-Solo-delincuentes_0_347965371.html> (26 nov. 2016).

FERNÁNDEZ, Pedro; TABARES, Eulalia. Homicidios en Venezuela. Sentido e impacto del uso de armas de fuego en Venezuela. Caracas: Comisión presidencial para control de armas, municiones y desarme, 2012.p. 71-114<comisionpresidencialdesarme.gob.ve>.

GABALDÓN, Luis Gerardo. Entrenamiento, riesgo y disposición hacia el uso de la fuerza física por policías venezolanos: una comparación entre candidatos y funcionarios. Analogías del Comportamiento, Boletín de la Escuela de Psicología, Universidad Católica Andrés Bello, 2016 (en prensa).

GABALDÓN, Luis Gerardo. La coacción entre lo público y lo privado. Espacio Abierto, v. 24, n. 4, p. 5-15, 2015.

GABALDÓN, Luis Gerardo. Seguridad, policía y control ciudadano. Encuentro de Organizaciones Sociales. Caracas: Universidad CatólicaAndrés Bello, 2013.p. 232-238.

GABALDÓN, Luis Gerardo. Incertidumbre y uso de la fuerza en el trabajo policial. Revista Brasileira de Segurança Pública, v. 4, n. 1, p. 32-48, 2010.

GABALDÓN, Luis Gerardo. La criminología latinoamericana: temas, perspectivas y políticas públicas en el tránsito del milenio. Espacio Abierto, v. 19, n. 2, p. 255-272, 2010.

GABALDÓN, Luis Gerardo. Seguridad ciudadana, confianza pública y policía en Venezuela. Revista Venezolana de Economía y Ciencias Sociales, v. 13, n. 3, p. 87-98, 2007.

GABALDÓN,LuisGerardo.Policíaycontrolsocial,1900-1999: fragmentación,centralización y difusión en América latina. Capítulo Criminológico, v. 27, n. 3, p. 129-150, 1999.

GABALDÓN, Luis Gerardo. Control social y criminología. Caracas: Editorial Jurídica Venezolana, 1987.

GABALDÓN, Luis Gerardo; ANTILLANO, Andrés. Las reformas policiales en Irlanda del Norte y en Venezuela: una visión comparada. Capítulo Criminológico, v. 36, n. 3, p. 5-27, 2008.

GABALDÓN, Luis Gerardo; BECERRA Nicanora; RAMÍREZ, María Daniela; CHACÓN, Andrea. Desarrollo y perspectivas de los comités ciudadanos de control policial. In: Luis Gerardo Gabaldón; Yoana Monsalve Briceño (orgs.). Control social 
y seguridad: investigación para la política pública. Mérida: Universidad de Los Andes, 2014. p. 163-213.

GABALDÓN, Luis Gerardo; BENAVIDES, David; DA ROCHA, Mafalda. Encuesta de situaciones y percepción del uso de armas de fuego (Espuaf). Sentido e impacto del uso de armas de fuego en Venezuela. Caracas: Comisión Presidencial para Control de Armas, Municiones y Desarme, 2012. p. 115-167<www.comisionpresidencialdesarme. gob.ve> (26 nov. 2016).

GABALDÓN, Luis Gerardo; BENAVIDES, David; PARRA Yasmely. Victimización delictiva y percepción de la policía. In: Gabaldón, Luis Gerardo;Andrés Antillano(orgs.). La policía venezolana desarrollo institucional y perspectivas de reforma al inicio del tercer milenio. Caracas: Comisión para la Reforma Policial, 2007. Tomo I, p. 307-339. GARLAND, David. Castigo y sociedad moderna. México: Siglo XXI, 1999.

GARRIDO, Vicente; STANGELAND, Per; REDONDO Santiago. Principios de criminología. Valencia: Tirant Lo Blanch, 2001.

INE, Instituto Nacional de Estadística. Encuesta nacional de victimización y percepción de convivencia ciudadana. Caracas: Universidad Nacional Experimental de la Seguridad, 2013.

INE, Instituto Nacional de Estadística. Encuesta nacional de victimización y percepción de seguridad ciudadana. Caracas: Consejo Nacional de Prevención y Seguridad Ciudadana, 2010.

KATZ, Jack. Seductions of Crime. Nueva York: Basic Books, 1988.

LISKA, Allen E; YU, Jiang. Specifying and testing the threat hypothesis: police use of deadly force. In: Allen E. Liska (org.). Social threat and social control. Albany: State University of New York Press, 1992. p. 53-68.

MACHADO, Jesús E. Estudio de los Consejos Comunales en Venezuela. Caracas Centro Gumilla, $2008<w w w . g u m i l l a . o r g / f i l e s / d o c u m e n t o s / E s t u d i o-C o n s e j o s$ Comunales01.pdf>.

MPPS (Ministerio del Poder Popular para la Salud 2012), República Bolivariana de Venezuela, Anuario de Mortalidad 2012. Caracas, 2014. p. 435 <www.stopvih.org/ Anuario-de-mortalidad-Venezuela-2012.pdf $>$.

MPPS (Ministerio del Poder Popular para la Salud 2011). Venezuela, Anuario de mortalidad 2011. Caracas, 2014. p. 387 <www.bvs.gob.ve/anuario/Anuario2011.pdf>.

MPPS (Ministerio del Poder Popular para la Salud 2010). Venezuela, Anuario de mortalidad 2010. Caracas, 2014. p. 389 <www.bvs.gob.ve/anuario/Anuario2010.pdf>.

MONSALVE BRICEÑO, Yoana. Repercusiones del sistema de justicia en el castigo policial. Capítulo Criminológico, v. 34, n. 1, p. 5-32, 2006.

MONSALVE BRICEÑO, Yoana; GABALDÓN, Luis Gerardo. Seguridad ciudadana, planificación y discurso político en Venezuela. In: Luis Gerardo Gabaldón; Yoana Monsalve Briceño (orgs.). Control social y seguridad: investigación para la política pública. Mérida: Universidad de Los Andes, 2014. p. 61-85.

MONSALVE BRICEÑO, Yoana; MUNÉVAR, Sandy. La identificación de la seguridad ciudadana en los proyectos sociales: una exploración en Venezuela. In: Luis Gerardo Gabaldón; Yoana Monsalve Briceño (coord.). Control social y seguridad: investigación para la política pública. Mérida, Universidad de Los Andes, 2014. p. 281-310. 
OPS, Oficina Panamericana de la Salud. Informe mundial sobre la violencia y la salud. Resumen. Washington, 2002.

PROVEA, Programa de Educación Acción en Derechos Humanos. Situación de los derechos humanos en Venezuela, Informe enero-diciembre 2013. Derecho a la manifestación pacífica, $2013<$ https://www.derechos.org.ve/informe-annual/informeanual-enero-diciembre-2013> (18 mayo 2015).

PNUD. Seguridad ciudadana con rostro humano: diagnóstico y propuestas para América Latina. Informe regional de desarrollo humano 2013-2014. Nueva York: Naciones Unidas, 2013.

RED de Apoyo por la Justicia y la Paz. Informe sobre impunidad y administración de justicia en Venezuela, 2000-2009. Investigación documental. Patrones de violación a los Derechos Humanos y mecanismos de impunidad. Serie Cuadernos Pensar en Derechos Humanos, v. 12, Caracas, 2010. p. 44.

RIVERO, Mirtha. La rebelión de los náufragos. Caracas: Editorial Alfa, 2010.

SANJUÁN, Ana María. La crítica situación de seguridad ciudadana en Venezuela. Características, causalidades, políticas y desafíos. In: Carlos Basombrío (org.). ¿A dónde vamos? Análisis de políticas públicas de seguridad en América Latina. Washington: Wilson Center, 2013. p. 297-347.

VENEZUELA. Ley para el desarme y control de armas y municiones, Gaceta Oficial n. 40.190, 17 de junio de 2013, 2013a.

VENEZUELA. Plan de la Patria, Segundo Plan Socialista de Desarrollo Económico y Social de la Nación 2013-2019, Gaceta Oficial de la República Bolivariana de Venezuela, n. 6.118, Extraordinaria, 4 de diciembre de 2013, 2013b.

VENEZUELA. Ley del estatuto de la función policial, Gaceta Oficial de la República Bolivariana de Venezuela, n. 5.940, Extraordinaria, 7 de diciembre de 2009.

VENEZUELA. Decreto n. 5.895 con Rango, valor y fuerza de la ley orgánica del servicio de policía y del cuerpo de Policía Nacional Bolivariana. Gaceta Oficial de la República Bolivariana de Venezuela, n. 5.880, Extraordinaria, 9 de abril de 2008.

WORDEN, Robert E. The causes of police brutality: theory and evidence on police use of force. In: William Geller; Hans Toch (orgs.). Police violence. New Haven: Yale University Press, 1996. p. 23-51.

ZUBILLAGA, Verónica. Los varones y sus clamores: los sentidos de la demanda de respeto y la lógica de la violencia entre jóvenes. Espacio Abierto, v. 16, n. 3, p. 577-608, 2007.

Autor correspondiente:

Luis Gerardo Gabaldón

Instituto de Investigaciones Jurídicas

Edificio de Posgrado, nivel 2

Universidad Católica Andrés Bello

Urbanización Montalbán, La Vega

Caracas 1020, Venezuela

Recibido el: 6 jun. 2016.

Aceptado el: 7 nov. 2016. 